\title{
PARP inhibitors as a radiosensitizer: a future promising approach in prostate cancer?
}

\author{
Martin Angel ${ }^{1, a}$ (iD, Martin Zarba² and Juan Pablo Sade ${ }^{3}$ \\ ${ }^{1}$ Medical Oncologist, Genitourinary Tumors Department, Instituto Alexander Fleming, Cramer 1180, Ciudad Autonoma de Buenos Aires, C1426ANZ, Argentina \\ ${ }_{2}^{2}$ Medical Oncology Fellow, FUCA, Cramer 1180, Ciudad Autonoma de Buenos Aires, C1426ANZ, Argentina \\ ${ }^{3}$ Medical Oncologist, Chief Genitourinary Tumors Department, Instituto Alexander Fleming, Cramer 1180, Ciudad Autonoma de Buenos Aires, \\ C1426ANZ, Argentina \\ ahttps://orcid.org/0000-0002-1463-8887
}

\begin{abstract}
Poly (ADP-ribose) polymerase (PARP) inhibitors (iPARPs) have shown efficacy in homologous recombination (HR) deficiency patients with advanced castration resistant prostate cancer and have shown a radiosensitizing effect in preclinical and early clinical trials. Preclinical data in prostate cancer cells suggest a similar cytotoxic effect with half the radiation dose under the effect of Olaparib or Rucaparib irrespective of HR status. Due to the biologic synergy of radiotherapy (RT) and iPARPs, the risk of recurrence of high-risk prostate cancer and the morbidity associated with prostate cancer local treatment, this interesting strategy seems promising, and a better understanding of the clinical implications remains to be elucidated.
\end{abstract}

Keywords: personalized medicine, radiosensitivity, target therapy

Poly(ADP-ribose) polymerase (PARP) is a DNA repair enzyme which has gained focus in the last decade due to the development of specific inhibitors (iPARPs) [1, 2]. The main role of PARP is to detect single-strand DNA breaks (SSB) and provide specific signals to trigger the enzymatic machinery involved in the SSB repair.

iPARP antitumor activity is based on the concept of synthetic lethality, in which two separate molecular pathways, that are not lethal when disrupted individually, cause cell death when inhibited simultaneously [3-6]. This molecular rational was translated to the clinic since iPARPs showed meaningful benefit in different tumor types, leading to FDA approval for the treatment of advanced ovarian, breast, and more recently, prostate cancer [7-11].

Interestingly, when combined with ionizing radiation, iPARP inhibition has been found to enhance cellular radiosensitivity [12-15]. Radiation induces physical and biochemical DNA damage and cells activate three main repair mechanisms. Two double-stranded DNA breaks (DSB) repair pathways ((non-homologous end-joining, (NHEJ), and homologous recombination, (HR)), and a single-stranded DNA breaks (SSB) repair pathway (base excision repair, BER) $[15,16]$.

In contrast to the more easily repaired SSB, DSB are highly mutagenic and cytotoxic if unrepaired. However, SSB can be converted to DSB in the context of DNA replication if unrepaired, interfering with important cellular processes and survival. Importantly, SSB
Correspondence to: Martin Angel

Email: mangel@alexanderfleming.org

ecancer 2021, 15:ed118

https://doi.org/10.3332/ecancer.2021.ed118

Published: $13 / 12 / 2021$

Received: 11/05/2021

Publication costs for this article were supported by ecancer (UK Charity number 1176307).

Copyright: (c) the authors; licensee ecancermedicalscience. This is an Open Access article distributed under the terms of the Creative Commons Attribution License (http:// creativecommons.org/licenses/by/4.0), which permits unrestricted use, distribution, and reproduction in any medium, provided the original work is properly cited. 
are more common in the context of external beam radiotherapy. Therefore, emerging evidence indicates that iPARP can act as radiosensitizers through the BER pathway, increasing the risk of collapsed replication forks, and thus generating persistent DSBs [17].

Furthermore, studies have showed that in addition to HR and NHEJ, DSBs may also be repaired by an alternative end-joining pathway (Alt-EJ), which requires PARP1 [18-20]. Consequently, iPARP use in cells that have switched to Alt-EJ, has demonstrated to enhance radiosensitivity [21].

BRCA1 and BRCA2 proteins are involved in HR repair. The accumulation of SSB that occurs when base excision repair (BER) is malfunctioning, i.e., when PARP is inhibited, produces an increase in DSB due to a failed replication fork that encounters a SSB [22]. These DSBs depend on BRCA proteins to proceed with a DNA conservative repair method (HR). When the BER and HR pathways are inaccessible to cells, they rely on a DNA non-conservative repair mechanism (NHEJ), which lead to genomic instability and cell death [23, 24].

Several iPARP has been investigated in preclinical studies [25]. An in vitro experiment showed a significant increase in DNA damage with the combination of the iPARP olaparib and rucaparib and X-radiation in a neuroblastoma model. Of note, the addition of iPARP allowed to decrease the radiation dose up to 50\% without losing its cytotoxic effect [26]. Rae et al, studied the effects of the same iPARPs in combination with radiation in prostate cancer cells. They found a similar cytotoxic effect with half of radiation dose when the cells were exposed to iPARP on both androgen dependent and androgen resistant lines [27, 28].

Clinical evidence on this novel investigational approach consists mainly in phase 1 studies. The first clinical trial exploring combination iPARP and ionizing radiation combines veliparib and whole brain radiation therapy (WBRT) in patients with brain metastases from non-small cell lung cancer. The combination therapy significantly prolongs survival in patient populations with favorable prognosis, but not in patients with unfavorable prognosis. However this benefit was not translated in the phase II trial [14, 29, 30].

In a phase I study of patients with stage II or III rectal cancer, patients were given neoadjuvant capecitabine and radiotherapy in conjunction with escalating doses of veliparib. Maximum tolerated dose was not reached, and the study found $400 \mathrm{mg}$ twice daily to be the appropriate dose of veliparib [31]. The phase II trial showed numerically more patients achieved a pathologic complete response with veliparib (22\% vs $34 \%$ ).

Recently, a phase $1 / 2$ study evaluated the safety of the combination of Olaparib with the radionucleide Ra-223 in pretreated patients with metastatic castrate resistant prostate cancer. The treatment was well tolerated and showed a 6-month rPFS (radiographic progression free survival) of 57\%. A phase 2 study is currently recruiting to frther test the hypothesis [32].

High-risk localized prostate cancer is managed with curative intent with surgery or with the combination of radiation therapy plus androgen deprivation therapy. However, approximately half of the patients experience recurrence, with significant prostate cancer-specific morbidity and mortality [33, 34]. Thus, for this group of patients, new treatment options are needed. Recent evidence suggests the existence of a close crosstalk between DNA damage response machinery and androgen hormone signaling pathways mainly based on exposure to ionizing radiation. The AR pathway is activated and transcriptionally upregulates a large subset of DNA repair genes, which improves DNA repair capacity and therefore promotes radio resistance of prostate cancer cells.

To date, scarce clinical evidence supports the benefit of combining iPARP and radiation therapy. This approach may be attractive for different tumor models, including high risk localized prostate cancer which is possibly one of the most suitable scenarios. In this setting, promising phase II trials are on-going, such as the NADIR study investigating niraparib with standard combination radiation therapy and androgen deprivation therapy in patients with high-risk prostate cancer (NCT04037254).

If this first experience shows promising results, it may be the beginning of a new strategy to treat high-risk localized prostate cancer. Currently, this strategy is based on the escalation of radiotherapy doses, on a longer time of ADT and on the intensification of the androgen blockade. Although all these maneuvers have been shown to be more effective, they entail a significant increase in the toxicity associated with the treatment.

\section{Conclusion}

While molecular alterations are currently being explored to make prostate cancer treatment more personalized, defects in DNA repair proteins are rare in localized prostate cancer. Since the potentiation of PARP inhibitors with RT is not based on these germinal or somatic molecular alterations, but on the mechanism of action of RT and ADT, they would be applicable to the majority of patients. To date, this interesting strategy seems promising, and a better understanding of the clinical implications remains to be elucidated. 


\section{Conflicts of interest}

None of the authors have conflicts of interest to declare.

\section{Funding}

The authors have not declared a specific grant for this research from any funding agency in the public, commercial or not-for-profit sectors.

\section{References}

1. Jannetti SA, Zeglis BM, and Zalutsky MR, et al (2020) Poly(ADP-ribose) polymerase (PARP) inhibitors and radiation therapy Front Pharmacol 11170

2. Herceg $Z$ and Wang ZQ (2001) Functions of poly(ADP-ribose) polymerase (PARP) in DNA repair, genomic integrity and cell death Mutat Res Mol Mech Mutagen 477(1-2) 97-110

3. Hartwell LH, Szankasi P, and Roberts CJ, et al (1997) Integrating genetic approaches into the discovery of anticancer drugs Science 278(5340) 1064-1068

4. Kaelin WG (2005) The concept of synthetic lethality in the context of anticancer therapy Nat Rev Cancer 5(9) 689-698

5. Kelland LR, Edwards SM, and Steel GG (1988) Induction and rejoining of DNA double-strand breaks in human cervix carcinoma cell lines of differing radiosensitivity Radiat Res 116(3) 526

6. Rehman FL, Lord CJ, and Ashworth A (2010) Synthetic lethal approaches to breast cancer therapy Nat Rev Clin Oncol 7(12) 718-724

7. de Bono J, Mateo J, and Fizazi K, et al (2020) Olaparib for metastatic castration-resistant prostate cancer N Engl J Med 382(22) 2091-2102

8. González-Martín A, Pothuri B, and Vergote I, et al (2019) Niraparib in Patients with Newly Diagnosed Advanced Ovarian Cancer N Engl $J$ Med 381(25) 2391-2402

9. Litton JK, Rugo HS, and Ettl J, et al (2018) Talazoparib in patients with advanced breast cancer and a germline BRCA mutation N Engl J Med 379(8) 753-763

10. Moore K, Colombo N, and Scambia G, et al (2018) Maintenance olaparib in patients with newly diagnosed advanced ovarian cancer N Engl J Med 379(26) 2495-2505

11. Robson M, Im SA, and Senkus E, et al (2017) Olaparib for metastatic breast cancer in patients with a germline BRCA mutation N Engl J Med 377(6) 523-533

12. Powell C, Mikropoulos C, and Kaye SB, et al (2010) Pre-clinical and clinical evaluation of PARP inhibitors as tumour-specific radiosensitisers Cancer Treat Rev 36(7) 566-575

13. Senra JM, Telfer BA, and Cherry KE, et al (2011) Inhibition of PARP-1 by olaparib (AZD2281) increases the radiosensitivity of a lung tumor xenograft Mol Cancer Ther 10(10) 1949-1958

14. Weigert $\mathrm{V}$, Jost $\mathrm{T}$, and Hecht $\mathrm{M}$, et al (2020) PARP inhibitors combined with ionizing radiation induce different effects in melanoma cells and healthy fibroblasts BMC Cancer 20(1) 775

15. Qiu Z, Oleinick NL, and Zhang J (2018) ATR/CHK1 inhibitors and cancer therapy Radiother Oncol 126(3) 450-464

16. Campistol JM, Sol M, and Rodriguez R, et al (1992) In vitro spontaneous synthesis of 2-microglobulin amyloid fibrils in peripheral blood mononuclear cell culture Am J Pathol 141(1) 7 
17. Dungey FA, Löser DA, and Chalmers AJ (2008) Replication-dependent radiosensitization of human glioma cells by inhibition of poly(ADP-ribose) polymerase: mechanisms and therapeutic potential Int J Radiat Oncol 72(4) 1188-1197

18. Jia G, Fu Y, and He C (2013) Reversible RNA adenosine methylation in biological regulation Trends Genet 29(2) 108-115

19. Mansour WY, Rhein T, and Dahm-Daphi J (2010) The alternative end-joining pathway for repair of DNA double-strand breaks requires PARP1 but is not dependent upon microhomologies Nucleic Acids Res 38(18) 6065-6077

20. Wang M, Wu W, and Wu W, et al (2006) PARP-1 and Ku compete for repair of DNA double strand breaks by distinct NHEJ pathways Nucleic Acids Res 34(21) 6170-6182

21. Toulany M (2019) Targeting DNA double-strand break repair pathways to improve radiotherapy response Genes 10 (1) 25

22. Farmer H, McCabe N, and Lord CJ, et al (2005) Targeting the DNA repair defect in BRCA mutant cells as a therapeutic strategy Nature 434(7035) 917-921

23. Tutt $A$ (2001) Mutation in Brca2 stimulates error-prone homology-directed repair of DNA double-strand breaks occurring between repeated sequences EMBO J 20(17) 4704-4716

24. Moynahan ME, Pierce AJ, and Jasin M (2001) BRCA2 is required for homology-directed repair of chromosomal breaks Mol Cell 7(2) 263-272

25. Fok JHL, Ramos-Montoya A, and Vazquez-Chantada M, et al (2019) AZD7648 is a potent and selective DNA-PK inhibitor that enhances radiation, chemotherapy and olaparib activity Nat Commun 10(1) 5065

26. Nile DL, Rae C, and Hyndman IJ, et al (2016) An evaluation in vitro of PARP-1 inhibitors, rucaparib and olaparib, as radiosensitisers for the treatment of neuroblastoma BMC Cancer 16(1) 621

27. Rae C and Mairs RJ (2017) Evaluation of the radiosensitizing potency of chemotherapeutic agents in prostate cancer cells Int J Radiat Biol 93(2) 194-203

28. Lesueur P, Lequesne J, and Grellard J-M, et al (2019) Phase I/Ila study of concomitant radiotherapy with olaparib and temozolomide in unresectable or partially resectable glioblastoma: OLA-TMZ-RTE-01 trial protocol BMC Cancer 19(1) 198

29. Mehta MP, Wang D, and Wang F, et al (2015) Veliparib in combination with whole brain radiation therapy in patients with brain metastases: results of a phase 1 study J Neurooncol 122(2) 409-417

30. Chabot P, Hsia TC, and Ryu JS, et al (2017) Veliparib in combination with whole-brain radiation therapy for patients with brain metastases from non-small cell lung cancer: results of a randomized, global, placebo-controlled study J Neurooncol 131(1) 105-115

31. Czito BG, Deming DA, and Jameson GS, et al (2017) Safety and tolerability of veliparib combined with capecitabine plus radiotherapy in patients with locally advanced rectal cancer: a phase 1b study Lancet Gastroenterol Hepatol 2(6) 418-426

32. Rana McKay R, Xie W, and Ajmera A, et al (2021) A phase 1/2 study of olaparib and radium-223 in men with metastatic castrationresistant prostate cancer (MCRPC) with bone metastases (COMRADE): results of the phase 1 study [Internet] J Clin Oncol 39(15_suppl) https://doi.org/10.1200/JCO.2021.39.15_suppl.e17020

33. Hoffman RM, Koyama T, and Fan KH, et al (2013) Mortality after radical prostatectomy or external beam radiotherapy for localized prostate cancer JNCI J Natl Cancer Inst 105(10) 711-718

34. Alicikus ZA, Yamada Y, and Zhang Z, et al (2011) Ten-year outcomes of high-dose, intensity-modulated radiotherapy for localized prostate cancer Cancer 117(7) 1429-1437 